COMPTES

RENDUS

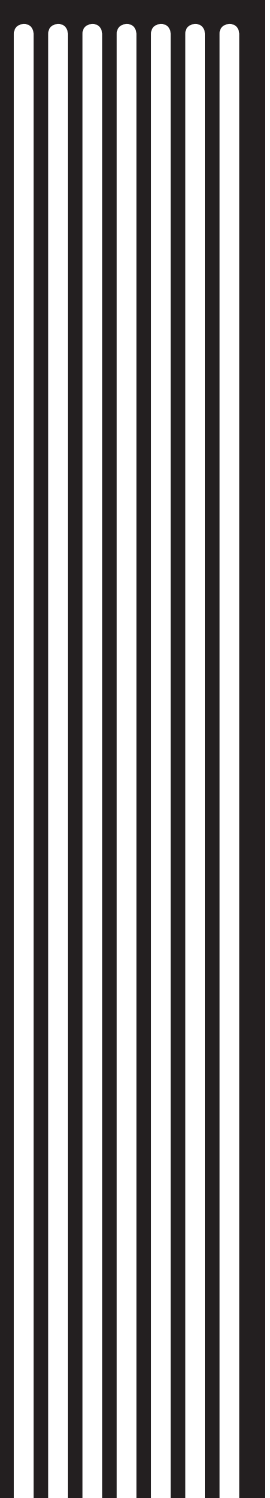



Établissement public de la Réunion des musées nationaux et du Grand Palais

des Champs-Élysées/Musée Hergé (ÉDs.)*

\section{Hergé, catalogue de l'exposition au Grand Palais}

Paris, Réunion des Musées Nationaux-Grand Palais/Bruxelles, Éditions Moulinsart 2016, 240 p.

SAMUEL BidAud [bidaudsamuel@gmail.com]

Univerzita Palackého v Olomouci, République tchèque

https://doi.org/10.5817/ERB2018-1-10

* Fe de erratas: este texto se imprimió en el número 38/2/2017 con un error de maquetación. Los editores le piden disculpas al autor.

C'est avec plaisir que les tintinophiles découvriront le catalogue de l'exposition «Hergé », qui s'est tenue du 28 septembre 2016 au 15 janvier 2017 au Grand Palais à Paris. Louvrage, richement illustré, est accompagné d'un certain nombre de textes qui se penchent sur différents aspects de l'œuvre d'Hergé, de Tintin à Jo, Zette et Jocko, en passant par la peinture à laquelle Hergé s'est discrètement consacré dans la dernière période de sa vie, à la fois comme peintre et comme collectionneur. C'est donc l'œuvre d'Hergé dans son intégralité et dans son évolution que le lecteur est invité à redécouvrir et à relire.

Les contributions à l'ouvrage sont variées. Dans l'Avant-propos, "L'immortalité comme l'un des Beaux-Arts » (pp. 25-27), Michel Serres (qui fut l'un des premiers à attirer l'attention des universitaires sur l'œuvre d'Hergé à l'époque et qui devint l'ami de ce dernier) revient sur le rapport à la création dans Les Aventures de Tintin, qui donnent à lire non seulement le beau dans les cases de chacun des albums, mais également "les luttes intimes " du créateur de Tintin et de " tout créateur au travail » (p. 25). Jérôme Neutres, dans son texte intitulé «Une fabrique de l'universel » (pp. 27-31), qui constitue également l'Introduction de l'ouvrage, se penche sur les raisons de l'universalité de Tintin, et retient notamment les suivantes : la « ligne claire» qui caractérise le dessin d'Hergé et la clarté narrative de son récit; le fait que tout le monde puisse s'identifier à Tintin, dont le visage est dépourvu de spécificité, qui est intemporel (Tintin ne vieillit pas), « hors sexe », et peut être lu par toutes les générations; et, enfin, les voyages, par lesquels Tintin explore les quatre coins du monde, de l'Écosse au Pérou. Mais l'art d'Hergé transcende tout cela, et si l'on peut parler d'un «monde de Tintin », c'est avant tout parce que « Hergé a créé un autre monde [...], avec son langage, ses pays, ses personnages récurrents et surtout son registre symbolique original » (p. 27). La contribution de Cécile Maisonneuve, « Hergé avec un grand A » (pp. 31-57), s'intéresse au rapport qu'Hergé entretint avec la peinture, et, plus spécifiquement, avec la création contemporaine, montrant comment les artistes admirés par Hergé font directement écho à son œuvre et à Tintin : «Des évidences apparaissent : Lichtenstein et Warhol, pour leur rapport direct à la bande dessinée ; Fontana dont les entailles subliment le trait ; Alechinsky pour la fulgurance de la ligne ; Herbin pour son alphabet plastique ; plus généralement, tout l'art minimaliste qui rejoint une esthétique de l'essentiel qui a toujours été la sienne [à Hergé] » (p. 35). Larticle de Benoît Mouchart, « Du dessin narratif avant toute chose " (pp. 57-87), se penche sur les caractéristiques du dessin dans Tintin, depuis les influences de Benjamin Rabier ou George McManus, qui ne furent jamais très profondes en ce qui concerne Hergé puisque ce dernier réinventa en partie le genre de la bande dessinée, à la collaboration avec Edgar P. Jacobs et Bob de Moor. Le style si caractéristique d'Hergé parvient, comme le souligne Benoît Mouchart, à donner l'illusion du temps et du mouvement, ce qui contribue à la fois à l'effet de réel et à l'universalité de Tintin : «Hergé dessine toujours davantage, dans ses premiers jets, l'idée d'une voiture en marche plutôt qu'un 
type de véhicule particulier. Ce sens exacerbé de la synthèse confère à sa représentation du monde une intemporalité universelle jamais démentie» (p. 60). Daniel Couvreur, dans son article « Comment Tintin a gagné la guerre » (pp. 87-111), revient sur l'attitude d'Hergé durant la Seconde Guerre Mondiale, depuis la disparition du journal Le Vingtième Siècle où Tintin était publié jusqu'à la participation d'Hergé au journal Le Soir, surnommé « Le Soir volé » et accusé de collaboration. L'auteur ne cache ni la caricature que fit Hergé dans L'Étoile mystérieuse du banquier juif américain Blumenstein, ni le drame et la violence de l'Épuration, qui marquera Hergé toute sa vie. Le texte de Jean-Marie Embs, « La foire aux solitudes» (pp. 111-139), s'intéresse à l'émergence progressive des personnages de Tintin, depuis les personnages des premiers albums qui apparaissent pour ne plus jamais revenir, à l'image de Coco dans Tintin au Congo, à la création de la famille de Tintin, avec le capitaine Haddock et le professeur Tournesol, jusqu'aux Bijoux de la Castafiore, testament d'Hergé, où « Moulinsart devient un microcosme où tous se croisent, s'entrechoquent, se méprennent dans un climat d'opacité, d'échec et de stérilité qui nous permet de mieux mesurer l'hermétisme de toutes les solitudes et bizarreries passées, celles, soudaines, d'un Alcazar, d'un Calys, celles, secrètes, d'un Saccharine, d'une concierge sentimentale ou émotive ou, tout simplement... de ces pékins qu'Hergé représente parfois, le regard étonné devant "l'autre", ce mystère impénétrable» (p. 115). Philippe Mello retrace, dans son étude « Cœurs Vaillants, Tintin, Jo, Zette et Jocko » (pp. 139-161), les rapports d'Hergé avec le journal Cours Vaillants, qui permit non seulement à Tintin d'atteindre le public français, mais qui fut également à l'origine de la création des Aventures de Jo, Zette et Jocko, lesquelles, selon le souhait de l'abbé Courtois qui dirigeait le journal, avaient pour but de montrer une famille unie, par opposition à Tintin où toute famille est absente. L'article de Dominique Maricq, « La publicité, une voie royale? » (pp. 161-181), s'intéresse à un aspect méconnu de l'œuvre d'Hergé, à savoir la publicité. Hergé, en effet, comme le rappelle Dominique Maricq, ne voyait au départ dans la bande dessinée qu'un divertis- sement, et envisagea d'abord de se consacrer à la publicité, au point de créer un Atelier Hergé-Publicité. Toutefois, «(d)evant le succès croissant et irrésistible des Aventures de Tintin, "Hergé, dessinateur publicitaire" se rend pourtant à lévidence : il faut céder la place à "Hergé, dessinateur de bandes dessinées”...» (p. 165). Pierre Baptiste se penche, dans son texte "Le "vent et l'os" » (pp. 181-205), sur la rencontre d'Hergé avec Tchang Tchong-jen et sur l'influence de ce dernier sur le dessin d'Hergé, notamment à travers deux des six principes formulés au VIème siècle par le théoricien chinois Xie He, que Tchang fait découvrir à Hergé : la nécessité de savoir saisir le souffle, "le fluide de la vie, ce qui fait qu'un oiseau, un arbre, une feuille ou un rocher sont ce qu'ils sont ", et la nécessité de peindre à partir de la «structure », de l'« ossature », ce qui constitue pour Hergé une «leçon du vide et du plein, des rapports possibles de composition entre les éléments dessinés » (p. 185). Philippe Goddin, enfin, dans son article «Aux sources de l'œuvre : de simples éclats de rire » (pp. 205-231), revient sur les débuts d'Hergé, de ses premiers dessins (son tout premier dessin, représentant un train qui passe devant une voiture et un garde-barrière, est reproduit p. 206) à Tintin au pays des Soviets, et évoque les influences de Benjamin Rabier, René Vincent, George McManus et Alain Saint-Ogan sur le jeune Hergé.

Louvrage se clôt par un "Cahier d'art pour les 7 à 77 ans " de 64 pages, réalisé par Jean-Michel Coblence. Chaque double page de ce cahier est consacrée à des thématiques variées accompagnées d'images des albums : "Le spectacle du monde", «Les lumières de la ville », «Visa pour la Syldavie » ou encore « Tintin au pied marin », pour n'en citer que quelques-unes.

Les illustrations de l'ouvrage, très nombreuses, font entrer dans la genèse de l'œuvre hergéenne. Des crayonnés de départ aux planches définitives, on découvre le labeur réalisé par Hergé pour chaque album et chaque case de Tintin ou des autres bandes dessinées qu'il a publiées, ainsi que d'anciennes pages de garde des albums, des feuillets de découpage de planches, des bleus de coloriage, des planches des albums en noir et blanc etc. Comme nous l'avons rappelé, l'intertexte hergéen est égale- 
ment exploré, depuis celui avec Alain Saint-Ogan et Zig et Puce, dont une planche qui montre ces derniers sollicités par la presse (p. 224) n’est pas sans rappeler les journalistes harcelant Tintin au début de Tintin au Congo pour avoir l'exclusivité de son reportage, à Mc Manus. Quant aux peintures d'Hergé reproduites dans l'ouvrage, qu'il s'agisse de celles qu'il a peintes ou de celles qu'il a acquises, si elles reflètent le goût d'Hergé pour l'art abstrait et semblent a priori contraster avec les voyages et l'aspect narratif de Tintin, elles rejoignent en même temps certaines thématiques hergéennes : on pense ainsi, devant Bleu de nuit de Stefan De Jaeger, qui montre un homme qui se dédouble et qui fut la dernière acquisition d'Hergé, à l'importance du double dans l'œuvre de ce dernier.

Peu d'œuvres nous accompagnent toute notre vie, de l'enfance à la vieillesse, mais c'est le cas de Tintin. Tintin est présent dans notre imaginaire. Il est, pour beaucoup d'entre nous, notre première lecture. Nous l'avons lu dès l'enfance, il est associé à nos souvenirs. Les albums de Tintin renvoient à une époque où il y avait encore des « lectures lentes ", où chaque case semblait receler un imaginaire infini. Qui n'a pas vogué avec Tintin au large de l'Afrique, en partance pour le Congo, ou n'est pas monté avec lui sur le pont du bateau qui le conduit en Égypte dans Les Cigares du pharaon? Qui ne s'est pas laissé baigner par la rêverie devant la mer couleur de nuit dans laquelle Tintin se laisse glisser au début du Temple du soleil pour monter à bord du Pachacamac, ou n'a pas été bercé par les images de Tintin cueillant des dattes dans le désert au crépuscule dans Au Pays de lor noir?

Nous pouvons alors conclure avec Jean-Marie Embs (p. 117) : « Hergé a disparu. Ses personnages restent. Les voici tous réunis devant nous sur cette fresque du Grand Palais. Alors, nous leur disons : Ainsi, vous êtes parfaits! Ne bougez surtout plus ! Tenez la pose! Gardons-les tous intacts, tels qu'ils furent, réunis ici pour une photo de famille et une incessante renaissance dans nos têtes ». 